\title{
Overlapping expression of microRNAs in human embry- onic colon and colorectal cancer
}

\author{
Mariano Monzo ${ }^{1,4, *}$, Alfons Navarro ${ }^{1,4, *}$, Eva Bandres ${ }^{2}$, Rosa Artells ${ }^{1,4}$, Isabel Moreno ${ }^{3}$, Bernat Gel ${ }^{1}$, Rafael Ibeas ${ }^{3}$, \\ Jose Moreno $^{3}$, Francisco Martinez ${ }^{3}$, Tania Diaz ${ }^{1}$, Antonio Martinez ${ }^{4,5}$, Olga Balagué, ${ }^{4,5}$, Jesus Garcia-Foncillas ${ }^{2}$ \\ ${ }^{1}$ Department of Human Anatomy and Embryology, School of Medicine, University of Barcelona, Barcelona, Spain; ${ }^{2}$ Laboratory of \\ Pharmacogenomics, Center for Applied Medical Research, University of Navarra, Spain; ${ }^{3}$ Department of Medical Oncology and \\ Surgery, Hospital Municipal de Badalona, Badalona, Spain; ${ }^{4}$ Institut d'Investigacions Biomediques August Pi i Sunyer (IDIBAPS), \\ School of Medicine, University of Barcelona, Barcelona, Spain; ${ }^{5}$ Hematopathology Section, Laboratory of Pathology, Hospital Clinic, \\ Barcelona, Spain
}

MicroRNAs (miRNAs) are essential for regulating cell differentiation and maintaining the pluripotent state of stem cells. Although dysregulation of specific miRNAs has been associated with certain types of cancer, to date no evidence has linked miRNA expression in embryonic and tumor tissues. We assessed the expression of mature miRNAs in human embryonic colon tissue, and in colorectal cancer and paired normal colon tissue. Overlapping miRNA expression was detected between embryonic colonic mucosa and colorectal cancer. We have found that the miR-17-92 cluster and its target, E2F1, exhibit a similar pattern of expression in human colon development and colonic carcinogenesis, regulating cell proliferation in both cases. In situ hybridization confirmed the high level of expression of miR-17-5p in the crypt progenitor compartment. We conclude that miRNA pathways play a major role in both embryonic development and neoplastic transformation of the colonic epithelium.

Keywords: miRNA, colorectal cancer, development, miRNA in situ hybridization, miR-17-5p, cluster miR-17-92

Cell Research (2008) 18:823-833. doi: 10.1038/cr.2008.81; published online 8 July 2008

\section{Introduction}

Mature microRNAs (miRNAs) are small RNA molecules (21-25 nucleotides in length) that act as negative regulators of gene expression, either inhibiting mRNA by blocking its translation into protein or destroying it through RNA interference [1]. miRNAs are essential for stem cell division, cell fate determination and patterning in early and later stages of development, where they regulate cell differentiation and maintenance of the pluripotent cell state [2, $3]$. The sequences of many miRNAs are conserved between distantly related organisms, suggesting that these molecules participate in essential processes, including normal embry-

\footnotetext{
*These two authors contributed equally to this work. Correspondence: Mariano Monzo, Department of Human Anatomy and Embryology, School of Medicine, University of Barcelona, Casanovas 143, 08036 Barcelona, Spain.

Tel: +34-934021903; Fax: +34-934035263

E-mail:mmonzo@ub.edu

Received 2 March 2008; revised 26 March 2008; accepted 31 March 2008; published online 8 July 2008
}

onic development and carcinogenesis $[4,5]$.

In addition, dysregulation of specific miRNAs has been associated with certain types of cancer, where they may act as either oncogenes or tumor suppressors, depending on their target genes [6,7]. For example, miR-15a and miR-16.1 have been associated with chronic lymphocytic leukemia [7]. miR-21 and mir-17-5p are upregulated, while miR-143 and miR-145 are downregulated in colorectal cancer [8-11]. miR-21 has been shown to have an antiapoptotic effect in glioma and breast cancer $[12,13]$, and the let-7 family of miRNAs is a prognostic factor in lung cancer [6].

It is generally accepted that identical genetic information can be found in embryonic, adult and tumor cells from the same individual. Cancerous cells are often described as being de-differentiated and expressing embryonal antigens. The genetic changes leading to disease modify the properties of the cancer cell, making it more closely related in both morphology and function to a stem cell $[14,15]$. However, to date, no evidence has linked miRNA expres- 
sion in embryonic and tumor tissues. In order to shed light on the possibility of such a link, we assessed the expression of 156 mature miRNAs in colon tissue from eleven 7- to 12week human embryos and in 44 human colorectal samples (22 tumor samples and 22 paired normal tissue samples). We postulate that miRNA expression may modulate the expression of gene products of signaling pathways involved in both intestinal development and cancer.

\section{Results}

miRNA expression during human colon development

Morphological analysis of 7-week-old embryonic tissue revealed an absence of intestinal lumen in the center of the colon and the presence of undifferentiated cells surrounded by embryonic mesenchyma (Figure 1A-1C). At 9 weeks, the intestinal lumen can be observed, and cells have the
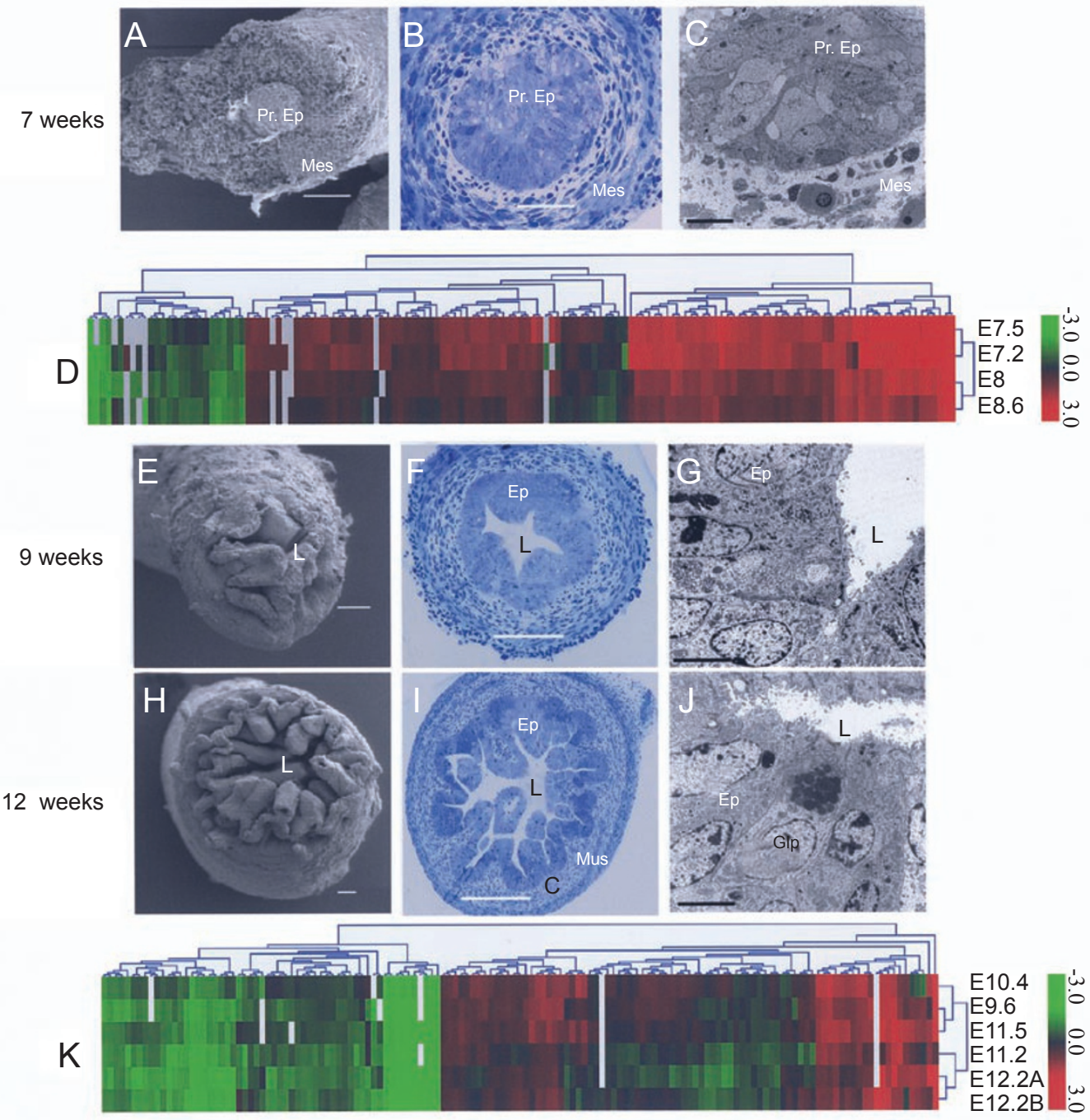

Figure 1 Morphology and hierarchical clustering of miRNA expression during the embryonic development of the human colon. (A-C) show the morphology of the human colon at 7 weeks: (A) by scanning electron microscope (SEM); (B) by semi-thin sections; and (C) by transmission electron microscope (TEM). At 7 weeks, the primitive epithelium (Pr. Ep) and the mesenchyme (Mes) are not structured. Figures (E-G) show the morphology of the human colon at 9 weeks: (E) by SEM; (F) by semi-thin sections; (G) by TEM. At 9 weeks, the intestinal lumen (L) begins to appear and the embryonic epithelium (Ep) can be seen. (H-J) show the morphology of the human colon at 12 weeks: (H) by SEM; (I) by semi-thin sections; (J) by TEM. At 12 weeks, the cells are completely differentiated into conjunctive tissue (C), muscular tissue (Mus) and epithelium (Ep), with the presence of Globet cells (Glo). (D and K) show the hierarchical clustering of miRNA expression at 7-8 and 9-12 weeks of development, respectively: (D) At 7-8 weeks, the majority of the miRNAs are overexpressed. (K) At 9-12 weeks, the expression levels of these miRNAs decrease (See Table 2 and Supplementary Table 2). The scale bars in the SEM images (A, E and $\mathbf{H}$ ) represent $50 \mu \mathrm{m}$; the scale bars in the semi-thin sections (B, $\mathbf{F}$ and $\mathbf{I})$ represent $100 \mu \mathrm{m}$; and the scale bars in the TEM images represent (C) $10 \mu \mathrm{m}$ and ( $\mathbf{G}$ and $\mathbf{J}) 5 \mu \mathrm{m}$. 
Table 1 Differential expression of miRNAs by SAM in embryonic human colon

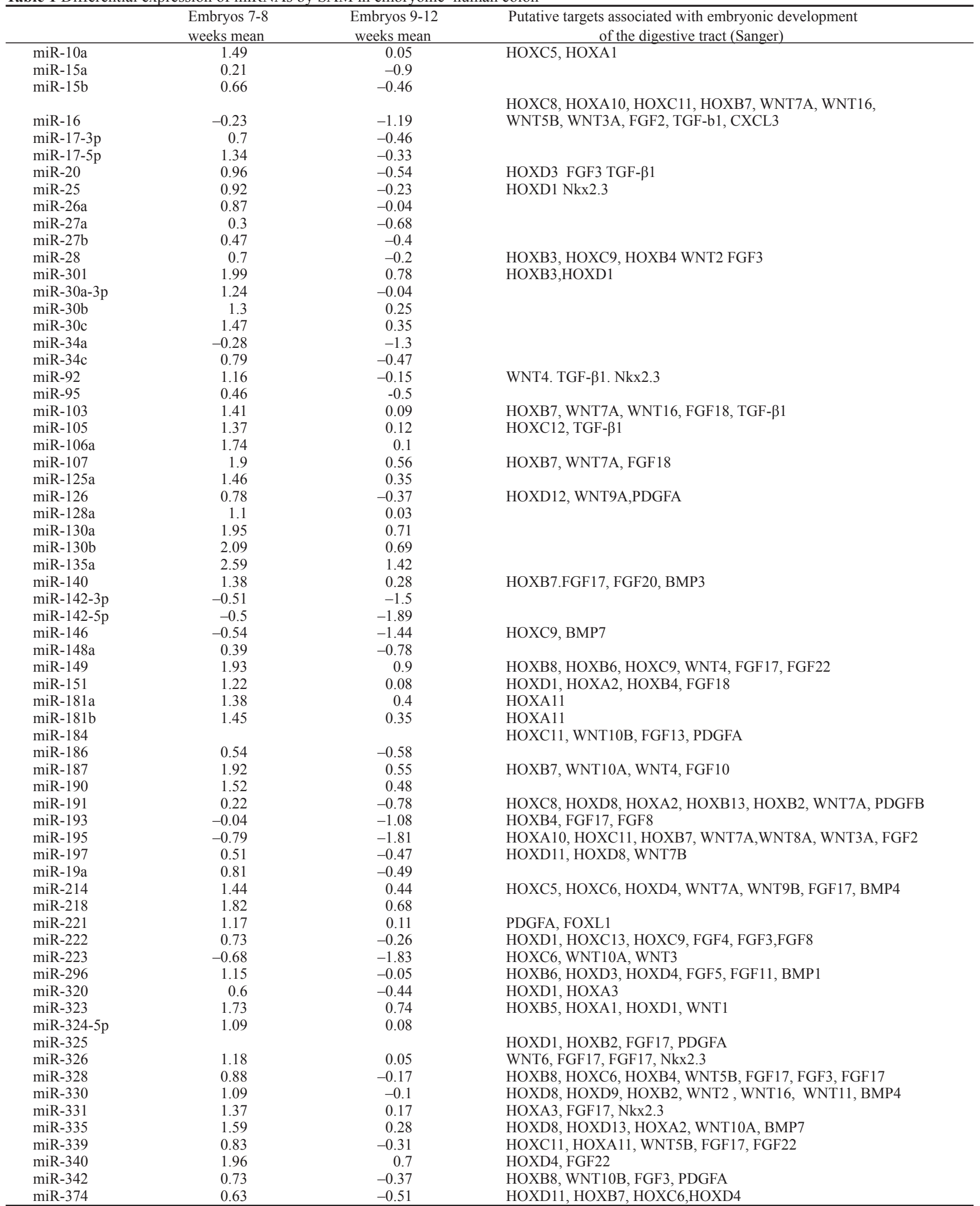

MicroRNAs differentially expressed in colon of 7-8-week vs 9-12-week embryos and their putative targets associated with embryonic development of the digestive tract. 
characteristics of columnar epithelium (Figure 1E-1G). At 12 weeks, the intestinal lumen is completely formed and intestinal epithelium is already differentiated, with the detectable presence of globet cells, mature fibroblasts and muscle tissue (Figure 1H-1J), as observed in previous studies [16].

In embryonic tissue, the analysis of 156 miRNAs by K-means revealed two well-differentiated groups: the embryos of 7-8 weeks and those of 9-12 weeks. At 7-8 weeks overall miRNA expression levels were high in comparison to normal adult colon tissue (shown in red in Figure 1D), while at 9-12 weeks expression levels were lower (shown in green in Figure 1K). SAM analysis identified 67 miRNAs differentially expressed between embryos at 7-8 weeks and those at 9-12 weeks (Supplementary information, Table S1). Forty of these miRNAs (59\%) are linked to putative targets (http://microrna.sanger.ac.uk) associated with the embryonic development of the digestive tract. miRNA expression decreased during epithelial differentiation
(Table 1).

Overlapping miRNA expression between embryonic and tumor colon tissues

When the expression of 156 miRNAs was compared among embryonic, tumor and normal tissues, overall low expression levels were observed in normal adult colon mucosa, while high levels were seen in embryonic tissue (Figure 2). Moreover, hierarchical cluster analysis identified four clear groups: colorectal cancer stage I (cluster 1), normal mucosa (cluster 2), embryonic tissue (cluster 3) and colorectal cancer stage II (cluster 4) (Figure 2). Lower miRNA expression was observed in cluster 1 , which included all stage $\mathrm{I}\left(\mathrm{T}_{2} \mathrm{~N}_{0} \mathrm{M}_{0}\right)$ and some stage II $\left(\mathrm{T}_{3} \mathrm{~N}_{0} \mathrm{M}_{0}\right)$ tumors, in comparison with cluster 4 , where all tumors were stage II $(p=0.014)$. No other clinical characteristics correlated with cluster 1 or cluster 4 (Figure 2 and Supplementary information, Table S2). The miRNA expression profile of stage I tumors was similar to that of normal tissue, while

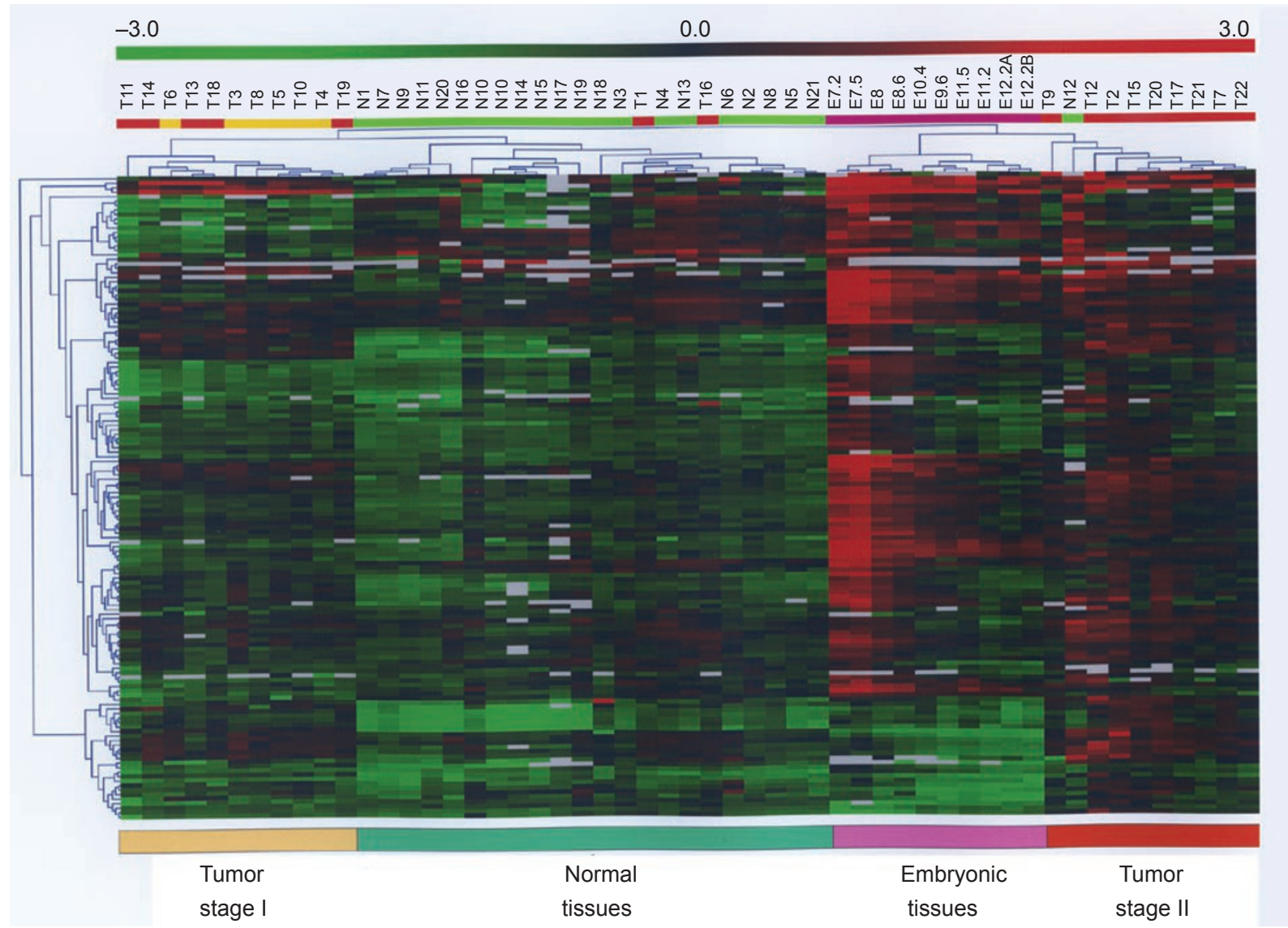

Figure 2 Hierarchical clustering of miRNAs in embryonic human colon and in adult colorectal cancer and paired normal colon tissue. The expression profiles observed in cluster 1 , which includes all the stage I tumors and some stage II tumors, are similar to those observed in cluster 2, which includes all the normal tissue. The expression profile of cluster 3 , which includes all the embryonic tissue, is similar to that of cluster 4 , which includes only stage II tumors. 
Table 2 Differentially regulated miRNA by SAM in stage I and stage II colorectal cancer tissue vs paired normal tissue.

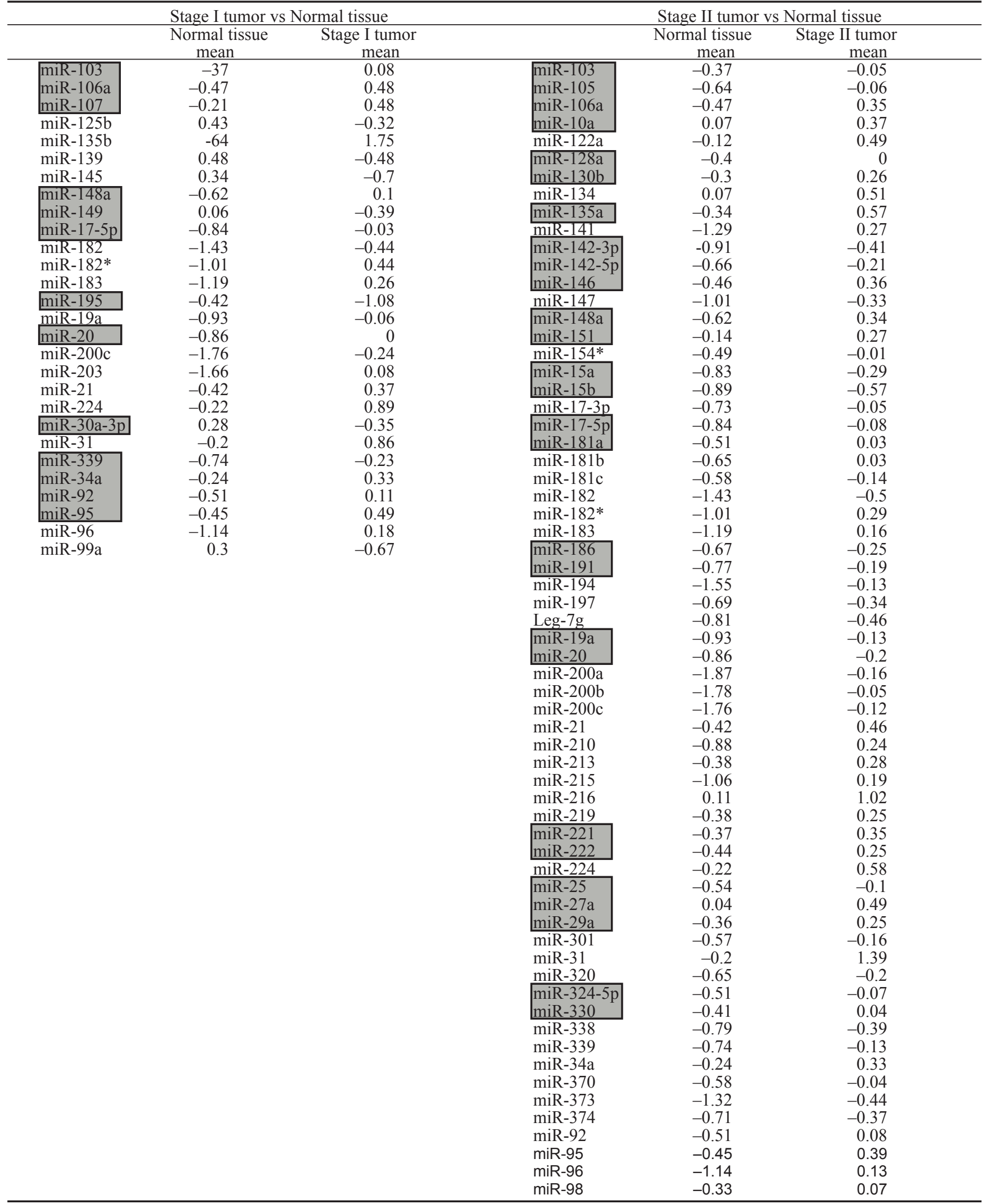

Twenty-eight miRNAs differentially expressed in stage I tumors vs normal tissue and 64 miRNAs expressed in stage II tumors vs normal tissue. The shaded miRNAs are also characteristic of early embryonic development. 
the expression profile of stage II tumors was closer to that of embryonic tissue.

Of the 156 miRNAs assessed, 13 were silenced in embryonic, tumor and normal colon tissues. Twenty-eight miRNAs were differentially expressed in stage I tumor tissue in comparison with paired normal tissue, and 13 of these 28 miRNAs (46\%) were also differentially expressed in tissue from 7-8-week embryos in comparison with 9-12-week embryos. Sixty-four miRNAs were differentially expressed in stage II tumor tissue in comparison with normal tissue, and 29 of these 64 miRNAs (45\%) were also differentially expressed in tissue from 7-8-week embryos in comparison with 9-12-week embryos (Table 2). These findings are similar to previous reports of miRNA dysregulation in colorectal cancer [17]. The miRNAs miR-17-5p, miR-191, miR-21, miR-107, miR-30c, miR-221 and miR-106a have been found to be upregulated in colorectal cancer tissue $[8,9]$, while expression of miR-145 is downregulated in colorectal cancer [18].

Cluster miR-17-92 modulates E2F1 expression and cell proliferation in human embryonic colon and colorectal cancer

SAM analysis identified three miRNAs (miR-17-5p,
miR-20 and miR-106a) that were differentially expressed among tumor stage I, tumor stage II and embryonic tissues versus normal colonic mucosa (Figure 3A). These miRNAs are members of the miR-17-92 cluster, supporting the idea that this cluster may play a role in human colon development and colorectal cancer growth. Moreover, it has been shown that $E 2 F 1$ expression is negatively regulated by the miR-17-92 cluster [19-21]. In the present study, E2F1 protein analysis revealed a pattern of expression opposite to that of the miR-17-92 cluster (Figure 3B). E2F1 protein expression was lower in the early stages of colon organogenesis and in carcinogenesis compared to normal mucosa (Figure 3C).

To test whether the miR-17-92 cluster regulates E2F1 expression in colon tissues, we used anti-miR-17-5p, antimiR-20 and anti-miR106a to inhibit miRNA function [12, 22]. Transfection with miR-17-5p, miR-20a and miR-106a antisense oligonucleotides (individual or pooled) showed that miR-17-5p is the critical member of the cluster involved in E2F1 expression regulation in colon cancer cells (Figure 3D). Interestingly, we found that anti-miR-17-5p reduced cell growth in a dose-dependent manner. At 25 $\mathrm{nM}$, growth inhibition by anti-miR-17-5p reached about
A
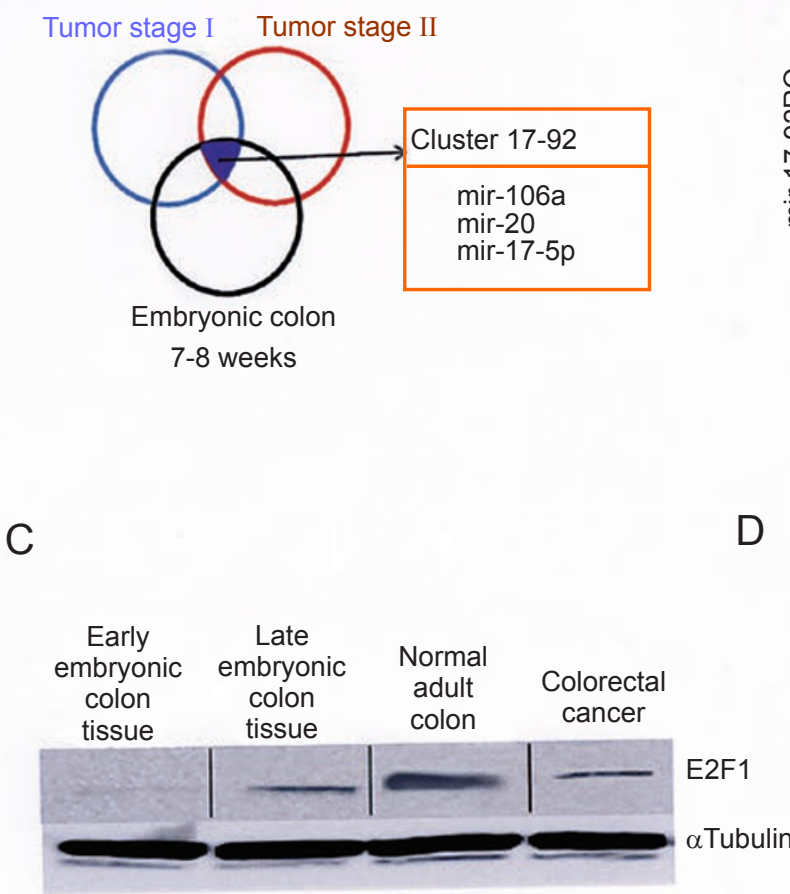

B
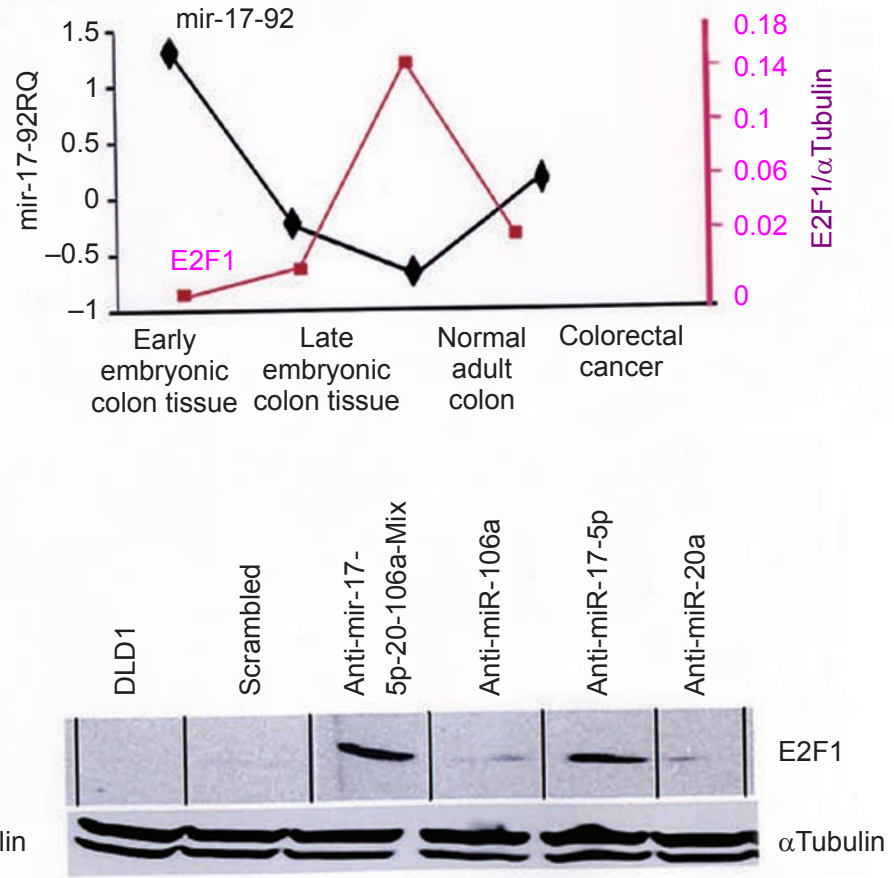

Figure 3 miR-17-5p modulates E2F1 protein expression in colon. (A) Venn diagram showing overlapping miRNAs between tumor and embryonic tissues. (B) Quantification of E2F1 protein (E2F1/ $\alpha$-tubulin) and the miR-17-92 cluster (mean Relative Quantification [RQ] of miR-17-5p, miR-106a and miR-20a) shows an opposing pattern of expression. (C) Western blot analysis confirmed that E2F1 is downregulated in the early process of colon organogenesis and carcinogenesis, while it is upregulated in normal colon tissue. (D) miR-17-5p inhibition in DLD1 colorectal cancer cells promotes E2F1 upregulation. 

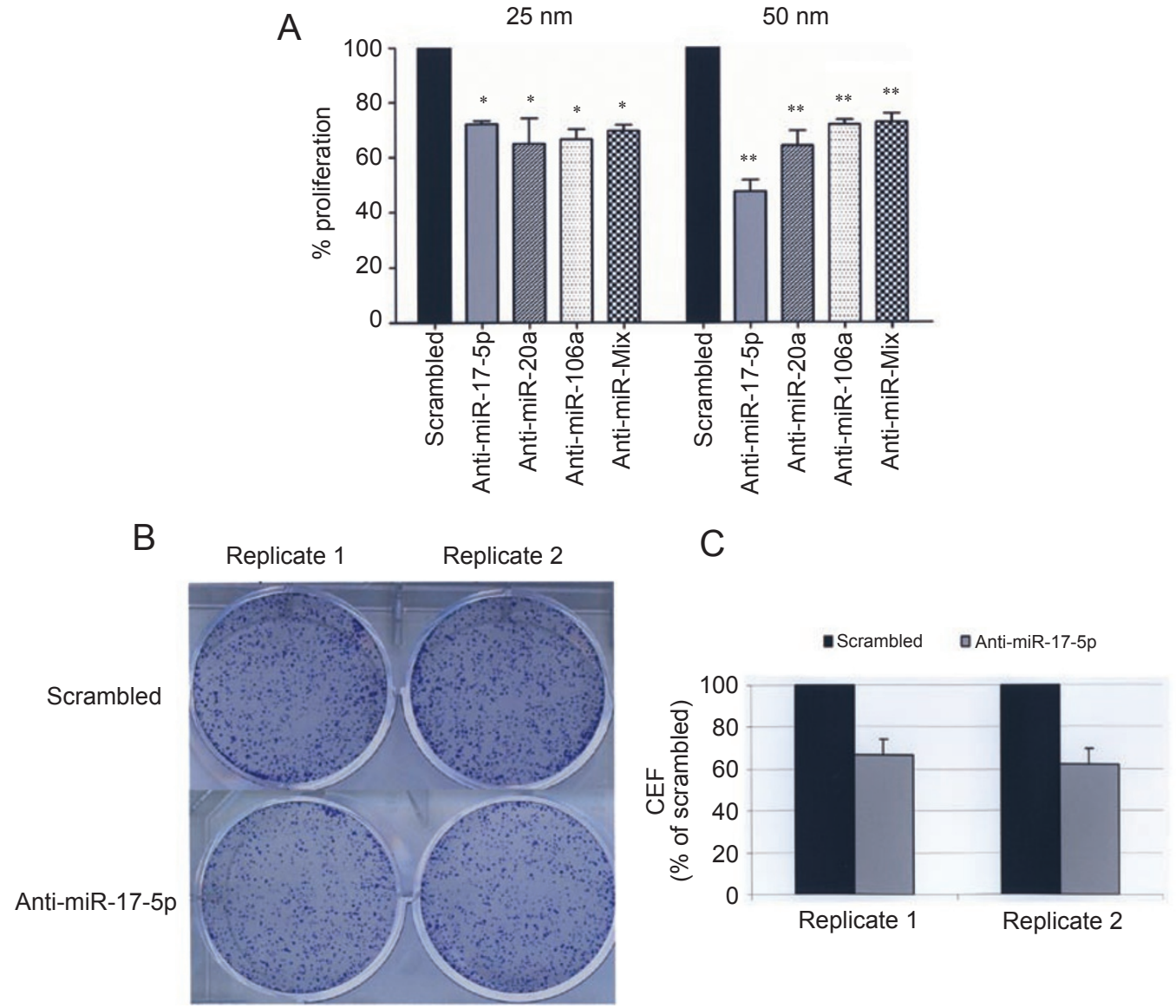

Figure 4 miR-17-5p regulates cell proliferation in colorectal cancer. (A) MTS proliferation assay results: percentage proliferation as compared to scrambled transfected cells 6 days after transfection with antisense oligonucleotides targeting miR-17-5p, miR-20a, miR106a, and anti-miR-Mix (pool of anti miR-17-5p, anti miR-20a and anti miR-106a). The data shown are the mean values \pm SD of three independent experiments. After $25 \mathrm{nM}$ transfection: miR-17-5p (72.05 $\pm 1.25 \%)$; miR-20a (64.85 $\pm 9.27 \%)$; miR-106a (66.41 $\pm 3.65 \%)$; miR-Mix (69.53 $\pm 2.04 \%)$. After $50 \mathrm{nM}$ transfection: miR-17-5p (47.35 $\pm 4.23 \%)$; miR-20a $(63.99 \pm 5.41 \%) ; \operatorname{miR}-106 \mathrm{a}(71.67 \pm 1.78 \%)$; miR-Mix (72.57 $\pm 3.17 \%) . * p<0.01, * * p<0.01$. (B) Two independent replicates (1 and 2) of DLD1 cells transfected with scrambled or miR-17-5p antisense were plated at low density (colony formation efficiency [CFE]); after 7 days they were fixed and stained with crystal violet. (C) Then, the dye was solubilized with methanol and OD was measured at $570 \mathrm{~nm}$. Lower CFE was seen in the cells transfected with anti-miR-17-5p. Data shown are the mean values \pm SD of five different OD: Replicate $1(66.6 \pm 7.3 \%)$; Replicate 2 $(61.8 \pm 2.1 \%)$.

$25 \%$, while reduction was more than $50 \%$ at $50 \mathrm{nM}$ (Figure $4 \mathrm{~A}$ ). Moreover, miR-17-5p-inhibited cells formed substantially fewer colonies than controls did when plated at low density (Figure 4B and 4C).

Chromogenic in situ hybridization of miR-17-5p in embryonic, tumor and normal colon tissue

Finally, to verify that miR-17-5p upregulation is associated with epithelial proliferative progenitor cells, a chromogenic in situ hybridization assay for miR-17-5p was performed in the embryonic tissue sections, as well as in paraffin-embedded paired normal and tumor samples. A clear miR-17-5p positive hybridization signal was present throughout the proliferative compartment in the base of the crypt, while its expression decreased along a gradient toward the top of the crypts (Figure 5A and 5B). In embryonic tissue, miR-17-5p was specifically expressed in early epithelium (endodermic tissue), whereas staining was not detected in the stromal compartment (mesodermic tissue) (Figure 5C). In tumor samples, miR-17-5p was expressed in tumor epithelium and in specific areas of the stromal compartment (Figure 5D).

\section{Discussion}

Recently, the role of several miRNAs in carcinogenesis 


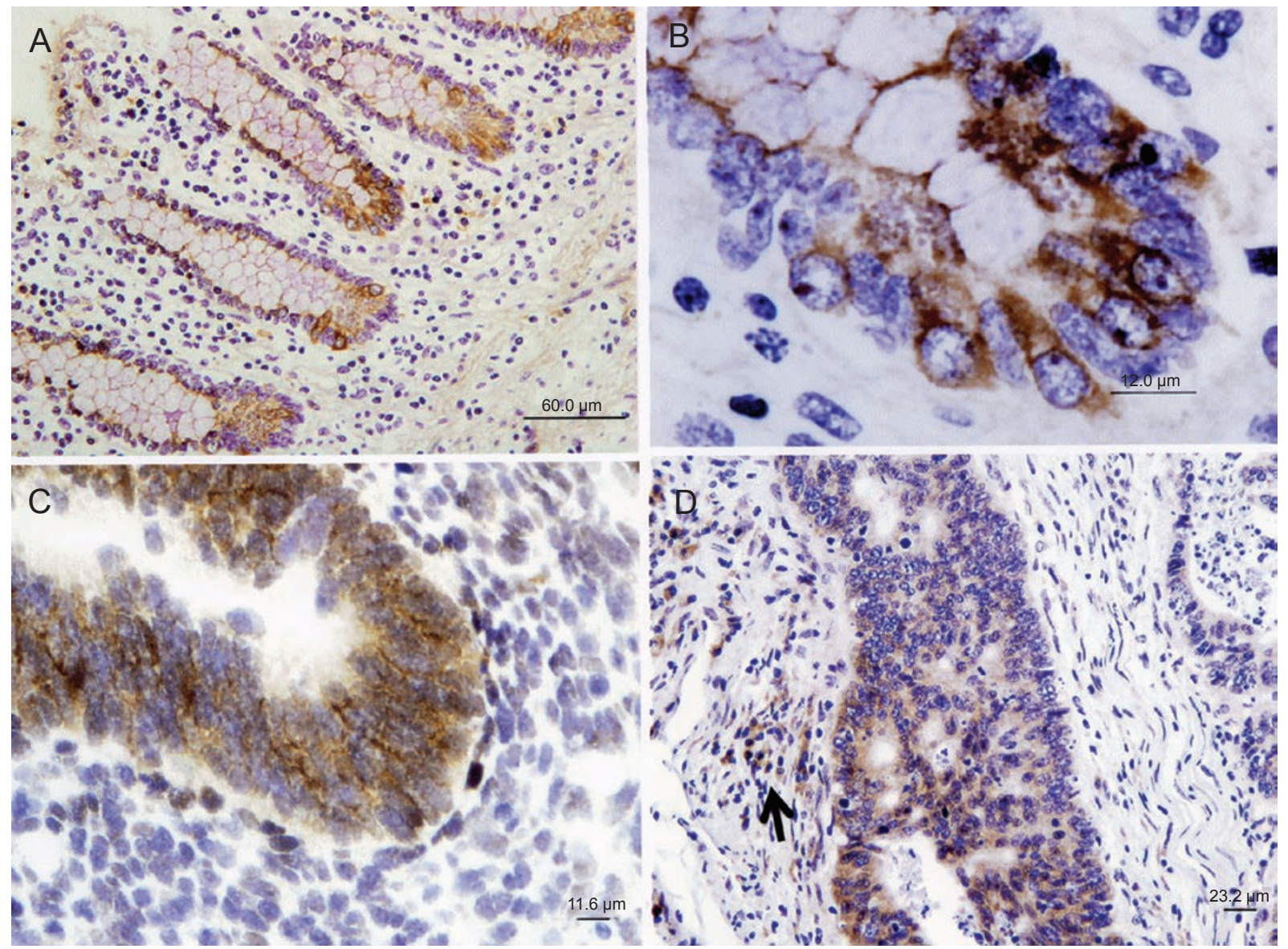

Figure 5 miRNA chromogenic in situ hybridization of miR-17-5p with LNA-ribo probe. (A) Normal colon tissue, showing detection of miR-17-5p in the base of the crypts. (B) Normal colon tissue, magnification of the crypt base showing high detection of the miR-17-5p at the proliferative compartment in the base of the crypt. (C) 10-week human embryonic colon, miR-17-5p is detected in mesodermic epithelium. (D) Tumor sample, miR-17-5p is detected in colorectal cancer cells and in some mesenchymal cells (arrows). The scale bar represents (A) $60 \mu \mathrm{m}$, (B) $12 \mu \mathrm{m}$, (C) $11.6 \mu \mathrm{m}$, (D) $23.2 \mu \mathrm{m}$.

and their impact on cancer prognosis have been described, but the relationship between the role of miRNAs in human embryogenesis and cancer remains to be clarified. The increased rate of proliferation and the lack of differentiation in cancer cells resemble what occurs in the physiological process of embryogenesis. In this study, we found that miRNAs that are expressed during the early development of the human colon are also active in the initial stages of colorectal cancer. Our findings support the hypothesis that miRNAs are highly expressed in undifferentiated cells and silenced during differentiation [3]. We observed that during the undifferentiated stage of human colon organogenesis (7-8 weeks) miRNA expression was high, but at the time differentiation of the epithelium occurred (9-12 weeks) miRNA expression decreased. In addition, the miRNA expression profile of stage I tumors was similar to that of normal tissue, while the expression profile of stage II tumors more closely resembled that of embryonic tissue. These results may reflect the fact that stage I tumors show an earlier phase of carcinogenesis with fewer genetic alterations and a more differentiated morphology, while stage II tumors may harbor a more aggressive phenotype with a greater capacity for proliferation, similar to that observed in embryonic tissues.

Overlapping miRNA expression was detected between embryogenesis and carcinogenesis. Of the shared miRNAs, the miRNA 17-92 cluster plays an important role in carcinogenesis and embryogenesis by regulating the proliferation process. The main effector of the cluster components was the miRNA miR-17-5p, through its target E2F1 [19]. Overexpression of the miR-17-92 cluster has been observed in B-cell lymphomas and lung cancer [23, 24] and inhibition of miR-17-5p selectively induces apoptosis in lung cancer cells [25]. Moreover, it has been shown that the miR-17-92 
cluster downregulates E2F1 in adult tissue [19], although it remains unclear whether the same target is modulated during development [26]. In the present study, E2F1 protein analysis in human embryonic colon and paired tumor and normal colon samples revealed a pattern of expression opposite to that of the miR-17-92 cluster. The downregulation of E2F1 expression by the miR-17-92 cluster during colon development may promote cell proliferation as part of the normal embryogenic process. In tumorigenesis, the downregulation of E2F1 expression by overexpression of the miR-17-92 cluster leads to an increased proliferation rate, probably due to the protection of tumor cells from apoptosis. In contrast, in normal adult colon tissue, low levels of miR-17-92 permit E2F1 expression. In the present study, we found that miR-17-5p was the crucial member of the miR-17-92 cluster. When cells were transfected with anti-miR-17-5p, E2F1 expression significantly increased, thus reducing cell proliferation. Even when anti-miR-17-5p was included in a pool with anti-miR-20 and anti-miR106a, which showed little or no effect on E2F1 expression, the impact of miR-17-5p was still significant, although somewhat diminished.

Colonic crypts offer an attractive model in which to study stem and progenitor cells, because these cells occupy discrete positions within crypts. Specifically, colonic crypt stem cells reside at the crypt base and give rise to a transient population of undifferentiated cells that vigorously proliferate as they migrate toward the lumen of the intestine [27]. miRNA in situ hybridization showed a miR-17-5p-positive signal throughout the proliferative compartment that gradually decreased toward the top of the crypts, suggesting a role in the homeostatic self-renewal of colon tissue. This result provides support for a potential role of miR-17-5p in controlling cell differentiation and proliferation in the large intestine.

Our findings demonstrate that miRNAs play an important role in colon organogenesis, where cell proliferation rates seem to be controlled by these miRNAs. In the normal adult colon these miRNAs are overexpressed only in the proliferation compartment, but in the tumor their expression increases throughout the proliferative zone.

In summary, the presence of a common miRNA expression pattern during human colon development and tumor growth supports the hypothesis of a relationship between carcinogenesis and embryogenesis [28]. Upregulation of the miR-17-92 cluster in the adult cell, when it had been silenced after organ development, could disrupt cell proliferation control. These findings provide a greater understanding of the mechanism of action of these miRNAs and, if validated in further experiments, will be a useful tool for the control of tumor growth, with a positive effect on future therapeutic applications [29].

\section{Materials and Methods}

\section{Human embryonic samples}

Eleven spontaneously aborted embryos and fetuses were donated with written informed consent to the Body Donation Service of the Human Anatomy and Embryology Department of the Hospital Clinic School of Medicine for morphological and molecular studies. The samples included large intestine from the seventh to the twelfth week of development. The large intestine samples (Supplementary information, Figure S1) were obtained using an Olympus stereo microscope SZ61. Samples for RNA extraction were preserved in liquid nitrogen.

\section{Patients}

Forty-four colorectal samples from 22 adult colorectal cancer patients were obtained through surgical resection between August 2002 and August 2004 at the Municipal Hospital of Badalona, Spain. Fifteen patients were male and seven female; six were stage I $\left(\mathrm{T}_{2} \mathrm{~N}_{0} \mathrm{M}_{0}\right)$ and 16 were stage II $\left(\mathrm{T}_{3} \mathrm{~N}_{0} \mathrm{M}_{0}\right)$ (Supplementary information, Table S2). All patients gave their signed informed consent. Tumor and paired normal fresh tissue samples were obtained from each patient and preserved in liquid nitrogen. RNA was obtained from the samples after histopathological confirmation of neoplastic and normal tissues.

\section{Scanning electron microscopy}

For scanning microscope analysis, the embryonic samples were fixed in a solution consisting of $2.5 \%$ glutaraldehyde in $0.1 \mathrm{M}$ phosphate buffer ( $\mathrm{pH} 7.2)$, post-fixed in osmium tetroxide (1\%) in the same phosphate buffer, dehydrated in graded alcohol and processed for critical point drying (Polaron). The samples were observed with a Zeiss DSM 940A operated at $10 \mathrm{kV}$.

\section{Transmission electron microscopy}

For transmission microscope analysis, the embryonic samples were fixed in a solution consisting of $2 \%$ paraformaldehyde and $2.5 \%$ glutaraldehyde in $0.1 \mathrm{M}$ phosphate buffer ( $\mathrm{pH} 7.2$ ), post-fixed in osmium tetroxide $(1 \%)$ in the same phosphate buffer, dehydrated in a graded acetone series and embedded in Spurr's resin. Thin sections (0.5-1 $\mu \mathrm{m})$ were used to select zones to explore through electron microscopy. Ultrathin sections $(60-80 \mathrm{~nm})$ were cut with an ultramicrotome (Leica UCT) using a diamond knife (Diatome). The ultrathin sections were mounted on $\mathrm{Cu}$ grids and post-stained, first with $2 \%$ uranyl acetate for $10 \mathrm{~min}$ and then with lead citrate for 20 min. Ultrastructural analysis was performed using a Jeol EM1010, operated at $80 \mathrm{kV}$ with a Bioscan 812 (Gatan) digital camera.

\section{$R N A$ extraction, reverse transcription and real-time $P C R$ quantification}

Total RNA was extracted from embryonic, tumor and normal tissues using Tripure total RNA isolation reagent (Roche Applied Science, Indianapolis, USA) as per the manufacturer's protocol. The concentration was quantified using GeneQuant Spectrophotometer (Pharmacia, Uppsala, Sweden). cDNA was synthesized from total RNA using gene-specific primers according to the TaqMan MicroRNA Assay protocol (PE Applied Biosystems, Foster City, CA). Reverse transcriptase reactions contained 10 ng of RNA samples, 50 nM stem-loop RT primer, $1 \times$ RT buffer, $0.25 \mathrm{mM}$ each of dNTPs, $3.33 \mathrm{U} / \mu 1$ MultiScribe reverse transcriptase and $0.25 \mathrm{U} / \mu \mathrm{l}$ RNase 
Inhibitor (all purchased from cDNA Archive kit of Applied Biosystems). The $7.5 \mu \mathrm{l}$ reactions were incubated in an Applied Biosystems 9500 ThermaCycler in a 96 -well plate for $30 \mathrm{~min}$ at $16{ }^{\circ} \mathrm{C}, 30 \mathrm{~min}$ at $42{ }^{\circ} \mathrm{C}, 5 \mathrm{~min}$ at $85^{\circ} \mathrm{C}$ and then held at $4{ }^{\circ} \mathrm{C}$. Real-time PCR was performed using an Applied Biosystems 7500 Sequence Detection system. The $10 \mu \mathrm{l}$ PCR included $0.67 \mu \mathrm{l}$ RT product, $1 \times$ TaqMan Universal PCR master mix and $1 \mu$ of primers and probe mix. The reactions were incubated in a 96 -well optical plate at $95{ }^{\circ} \mathrm{C}$ for 10 min, followed by 40 cycles of $95^{\circ} \mathrm{C}$ for $15 \mathrm{~s}$ and $60^{\circ} \mathrm{C}$ for $1 \mathrm{~min}$. The threshold cycle $(\mathrm{Ct})$ data were determined using default threshold settings. The $\mathrm{Ct}$ is defined as the fractional cycle number at which the fluorescence passes the fixed threshold.

\section{Normalization and data analysis}

miRNA expression data were normalized to let7-a miRNA, in accordance with our findings in previous studies, where we had observed that different approaches, including global median and $18 \mathrm{~S}$ RNA normalization, showed similar results [8]. Relative quantification of miRNA expression was calculated with the $2^{-\triangle \triangle \mathrm{Ct}}$ method (Applied Biosystems User Bulletin $\mathrm{N}^{\circ} 2$ [P/N 4303859]). The data were presented as $\log _{10}$ of relative quantity (RQ) of target miRNA, normalized in regard to miR-let-7a and relative to a calibrator sample. Normal human colorectal tissues were used as calibrators for all samples.

Data were analyzed using TIGR Multiexperiment viewer version 3.1 (Dana-Faber Cancer Institute, Boston USA). Data are presented as $\log _{10}$ of RQ of target miRNA relative to a control sample. To identify miRNAs with significantly differential expression levels between embryo, tumor and normal tissue samples, two multivariate permutation tests provided in TIGR Multiexperiment viewer were performed: Significance Analysis of Microarrays (SAM) and Student's $t$-test. In both cases, we selected paired $t$-test options to compare tumor versus normal tissue with an false discovery rate (FDR) of less than $10 \%$. Hierarchical clustering was performed with the normalized data using average linkage and Euclidean distance as measures of similarity.

Associations between tumor clusters and clinical variables were measured by Fisher's exact test on a $2 \times 2$ contingency table using SPSS 12.0.1 statistical software (1999, SPSS, Chicago, IL).

\section{Antisense inhibition of miR-17-92 cluster}

For the analysis of E2F1, 25 and 50 pmol each targeting mir-17-5p, mir-20a and mir-106 or 100 pmol of the scrambled oligonucleotide were transfected into DLD1 colorectal cancer cell lines growing in six-well dishes (plated at 200000 cells per well $24 \mathrm{~h}$ before transfection) using Lipofectamine 2000 . We confirmed transfection efficiency ( $>95 \%$ ) using Silencer FAM-labeled Negative Control (Ambion). Cell lysates were collected 24, 48 and $72 \mathrm{~h}$ after transfection; miRNA expression was analyzed by real-time PCR and E2F1 expression was assessed through immunoblotting.

\section{Cell proliferation assays}

For cell proliferation, $24 \mathrm{~h}$ after anti-miRNA transfection, cells were trypsinized, and 3000 viable cells were plated into 96-well plates or 5000 viable cells were plated in 6-well plates. Cell growth was measured with CellTiter 96 non-radioactive cell proliferation assay kit (Promega), counting was performed with the NucleoCounter Automated Cell Counting System (Chemometec), and cells were visualized following fixation and staining with crystal violet.

\section{Western immunoblotting}

Total protein from embryonic, tumoral and normal colon samples was isolated using Qiagen Qproteome Mammalian Protein Prep Kit according to the manufacturer's protocol, with minor modifications. Equal amounts of proteins $(50 \mu \mathrm{g})$ were separated by SDS-polyacrylamide electrophoresis in Precast Ready gels from Biorad (10\% Tris- $\mathrm{HCl}$ polyacrylamide), and transferred to pure nitrocellulose membranes (Trans-Blot Transfer Medium, Biorad). Membranes were incubated with mouse monoclonal antibodies against E2F1 (1:200, Santa Cruz Biotechnology, Santa Cruz, California) and $\alpha$-tubulin (1:5 000, Sigma, Saint Louis, Missouri). Antibody binding was revealed by incubation with anti-mouse IgG peroxidase conjugate secondary antibodies (Sigma, Saint Louis, Missouri). Chemiluminescence was detected using SuperSignal West Pico Chemiluminescent Substrate (Pierce Biotechnology, Rockford, IL) and read in Chemidoc System (Biorad). The protein density of the bands was quantified using the Quantity one software v 4.2.6 and the relative quantification was calculated with reference to $\alpha$-tubulin signal.

\section{miRNA in situ hybridization}

Fluorescein (FITC) 5' labeled locked-nuclei-acid-incorporated (LNA) miRNA ribo probes for miR-17-5p (miRCURY ${ }^{\mathrm{TM}}$ LNA detection, Exiqon) were used in formalin-fixed, paraffin-embedded tissue sections on silane-coated slides (Vision BioSystem). Chromogenic in situ hybridization was performed in a Bond Max (Vision Biosystems) automated platform. Pre-treatment of the slides was performed with Protease 1 for $10 \mathrm{~min}$ at $37^{\circ} \mathrm{C}$. A total amount of $300 \mu \mathrm{l}$ of 25 $\mathrm{nM}$ probe was hybridized in $1 \times$ sodium chloride-sodium citrate hybridization buffer (SSC) (Innogenetics) at up to $50{ }^{\circ} \mathrm{C}$ for $5 \mathrm{~h}$. We used a pre-diluted mouse anti-FITC antibody (Vision BioSystems) for 20-60 min, followed by a goat anti-mouse linked to thousands of horseradish peroxidase (HRP) sites (Refine Detection System, Vision BioSystems). DAB was used as a chromogen (reacted for 10 min) and hematoxylin was used as a counterstain.

\section{Acknowledgments}

We thank D Fuster, S Jansa and A Pons (University of Barcelona, Spain) for technical help, and Dr A Urbano (Hospital Virgen del Rocio, Spain) and R O'Brate for comments on the manuscript and language revision. We also thank Dr S Barambio, Y Trejo and R Lopez (Clinica d'Ara, Spain) for comments on embryology. This work was supported by grants from the SDCSD of the School of Medicine of the University of Barcelona, Fundacion Olga Torres, and Fondo Investigaciones Sanitarias de la Seguridad Social FIS-PI060087.

\section{References}

1 Kim VN, Nam JW. Genomics of microRNA. Trends Genet 2006; 22:165-173.

2 Hatfield SD, Shcherbata HR, Fischer KA, et al. Stem cell division is regulated by the microRNA pathway. Nature 2005; 435:974978.

3 Houbaviy HB, Murray MF, Sharp PA. Embryonic stem cell- 
specific MicroRNAs. Dev Cell 2003; 5:351-358.

4 Farh KK, Grimson A, Jan C, et al. The widespread impact of mammalian MicroRNAs on mRNA repression and evolution. Science 2005; 310:1817-1821.

5 Esquela-Kerscher A, Slack FJ. Oncomirs - microRNAs with a role in cancer. Nat Rev Cancer 2006; 6:259-269.

6 Eder M, Scherr M. MicroRNA and lung cancer. $N$ Engl J Med 2005; 352:2446-2448.

7 Calin GA, Ferracin M, Cimmino A, et al. A MicroRNA signature associated with prognosis and progression in chronic lymphocytic leukemia. N Engl J Med 2005; 353:1793-1801.

8 Bandres E, Cubedo E, Agirre X, et al. Identification by realtime PCR of 13 mature microRNAs differentially expressed in colorectal cancer and non-tumoral tissues. Mol Cancer 2006; 5:29.

9 Volinia S, Calin GA, Liu CG, et al. A microRNA expression signature of human solid tumors defines cancer gene targets. Proc Natl Acad Sci USA 2006; 103:2257-2261.

10 Lanza G, Ferracin M, Gafa R, et al. mRNA/microRNA gene expression profile in microsatellite unstable colorectal cancer. Mol Cancer 2007; 6:54.

11 Schetter AJ, Leung SY, Sohn JJ, et al. MicroRNA expression profiles associated with prognosis and therapeutic outcome in colon adenocarcinoma. JAMA 2008; 299:425-436.

12 Chan JA, Krichevsky AM, Kosik KS. MicroRNA-21 is an antiapoptotic factor in human glioblastoma cells. Cancer Res 2005; 65:6029-6033.

13 Iorio MV, Ferracin M, Liu CG, et al. MicroRNA gene expression deregulation in human breast cancer. Cancer Res 2005; 65:70657070 .

14 Lepourcelet M, Tou L, Cai L, et al. Insights into developmental mechanisms and cancers in the mammalian intestine derived from serial analysis of gene expression and study of the hepatomaderived growth factor (HDGF). Development 2005; 132:415427.

15 Kho AT, Zhao Q, Cai ZH, et al. Conserved mechanisms across development and tumorigenesis revealed by a mouse development perspective of human cancers. Genes Dev 2004; 18:629640.

16 Montgomery RK, Mulberg AE, Grand RJ. Development of the human gastrointestinal tract: twenty years of progress. Gastro- enterology 1999; 116:702-731.

17 Cummins JM, He YP, Leary RJ, et al. The colorectal microRNAome. Proc Natl Acad Sci USA 2006; 103:3687-3692.

18 Akao Y, Nakagawa Y, Naoe T. MicroRNA-143 and-145 in colon cancer. DNA Cell Biol 2007; 26:311-20.

19 O'donnell KA, Wentzel EA, Zeller KI, Dang CV, Mendell JT. c-Myc-regulated microRNAs modulate E2F1 expression. Nature 2005; 435:839-843.

20 Woods K, Thomson JM, Hammond SM. Direct regulation of an oncogenic micro-RNA cluster by E2F transcription factors. $J$ Biol Chem 2007; 282:2130-2134.

21 Novotny GW, Sonne SB, Nielsen JE, et al. Translational repression of E2F1 mRNA in carcinoma in situ and normal testis correlates with expression of the miR-17-92 cluster. Cell Death Differ 2007; 14:879-882.

22 Cheng AM, Byrom MW, Shelton J, Ford LP. Antisense inhibition of human miRNAs and indications for an involvement of miRNA in cell growth and apoptosis. Nucleic Acids Res 2005; 33:1290-1297.

23 He L, Thomson JM, Hemann MT, et al. A microRNA polycistron as a potential human oncogene. Nature 2005; 435:828-833.

24 Hayashita Y, Osada H, Tatematsu Y, et al. A polycistronic microRNA cluster, miR-17-92, is overexpressed in human lung cancers and enhances cell proliferation. Cancer Res 2005; 65:9628-9632.

25 Matsubara H, Takeuchi T, Nishikawa E, et al. Apoptosis induction by antisense oligonucleotides against miR-17-5p and miR-20a in lung cancers overexpressing miR-17-92. Oncogene 2007; 26:6099-6105.

26 Rajewsky N. microRNA target predictions in animals. Nat Genet 2006; 38 Suppl:S8-S13.

27 Potten CS. Stem cells in gastrointestinal epithelium: numbers, characteristics and death. Philos Trans R Soc Lond B Biol Sci 1998; 353:821-830.

$28 \mathrm{Hu} \mathrm{M}$, Shivdasani RA. Overlapping gene expression in fetal mouse intestine development and human colorectal cancer. Cancer Res 2005; 65:8715-8722.

29 Meng F, Henson R, Lang M, et al. Involvement of human micro-RNA in growth and response to chemotherapy in human cholangiocarcinoma cell lines. Gastroenterology 2006; 130:21132129.

(Supplementary Information is linked to the online version of the paper on the Cell Research website.) 